\title{
L'apprentissage lexical en deuxième langue : des réseaux évolutifs
}

\section{Peter Prince}

\section{Q OpenEdition}

\section{Journals}

\section{Édition électronique}

URL : http://journals.openedition.org/asp/2596

DOI : 10.4000/asp.2596

ISBN : 978-2-8218-0380-0

ISSN : 2108-6354

\section{Éditeur}

Groupe d'étude et de recherche en anglais de spécialité

Édition imprimée

Date de publication : 1 décembre 1999

Pagination : 335-347

ISSN : 1246-8185

\section{Référence électronique}

Peter Prince, "L'apprentissage lexical en deuxième langue : des réseaux évolutifs », ASp [En ligne], 23-26 | 1999, mis en ligne le 11 novembre 2011, consulté le 20 avril 2019. URL : http:// journals.openedition.org/asp/2596 ; DOI : 10.4000/asp.2596

Ce document a été généré automatiquement le 20 avril 2019.

Tous droits réservés 


\title{
L'apprentissage lexical en deuxième langue : des réseaux évolutifs
}

\author{
Peter Prince
}

\section{Introduction}

1 L'idée que nos représentations mentales trouvent un support anatomique sous forme de réseaux de neurones n'est pas nouvelle. Selon Hebb (1949), à chaque fois que deux neurones connectés se déchargent en même temps, le lien entre eux se renforce. La formation d'une représentation mentale, pour autant qu'elle possède des frontières fixes, implique de grands nombres de neurones, tissant des miniréseaux qui sont eux-mêmes interconnectés pour former des réseaux plus vastes. Bien que son mécanisme précis ne soit pas encore entièrement expliqué sur le plan neurophysiologique, le principe mis en avant par Hebb serait à la base de l'apprentissage, que Rose (1993) définit par ailleurs dans les termes suivants :

Learning is a response by an animal to a novel situation such that, when confronted subsequently with a comparable situation, the animal's behaviour is reliably modified in such a way that its response is more appropriate.

Proposée par un biologiste moléculaire, cette définition convient néanmoins à l'apprentissage lexical, où l'animal en question est l'homme. Prenons le cas d'un apprenant dont la première langue (L1) est le français et la langue cible (L2) l'anglais. En matière d'apprentissage lexical, un comportement approprié consiste, par exemple, à remarquer que window désigne non seulement la fenêtre d'une maison mais aussi le hublot d'un avion. En conséquence, la représentation conceptuelle correspondant à window est réaménagée en L2, ce qui permet à l'apprenant dans un premier temps de reconnaître le mot hublot, et dans un deuxième temps de le produire. Si une telle vision de l'évolution des représentations lexicales est compatible avec des données à la fois en L1 (Monsell 1991), et en L2 (de Groot 1992), il reste de nombreuses questions à éclaircir, dont deux sont centrales pour le présent article, à savoir comment se développe le lexique mental L2, et par quels moyens pédagogiques il est possible d'aider à son développement. 


\section{Du réseau de neurones au réseau de mots : l'apport du connexionnisme}

3 L'étude des phénomènes mentaux fait intervenir plusieurs niveaux d'analyse. Marr (1982) en distingue trois : le niveau du substrat anatomique, celui des algorithmes utilisés dans le traitement de l'information et celui des constructions issues de ce traitement. Le premier niveau intéresse la neurophysiologie et la notion de réseau de neurones y trouve une place centrale. Le deuxième niveau met l'accent sur les processus auxquels participent ces réseaux, et le troisième niveau fait encore appel à la notion de réseau, cette fois sous forme de réseaux de représentations, en l'occurrence lexicales. En linguistique, l'idée de réseau lexical est présente depuis longtemps puisqu'elle sous-tend celle de champ sémantique, introduit par Trier en 1931 (cité dans Bierwisch 1970) pour mettre en évidence, sinon pour formaliser, les rapports sémantiques existant entre les mots. Par ailleurs, elle se trouve aussi en arrière-plan dans beaucoup de travaux d'ordre psycholinguistique, où il est supposé que le lexique mental est organisé en réseau obéissant à la fois à des contraintes physiologiques et des propriétés linguistiques. S'agissant de ce troisième niveau d'analyse, cependant, la question de l'apprentissage est souvent laissée de côté dans la mesure où les liens entre représentations lexicales sont supposés être stables (par exemple chez l'adulte monolingue ou chez le bilingue avancé).

4 À partir des années 1980, le connexionisme, dépassant certaines tentatives infructueuses précédentes, met en avant l'idée que les représentations mentales sont distribuées à travers de grandes populations d'unités reliées entre elles par des connexions plus ou moins fortes (Rumelhart \& McClelland 1986). Cette conception remet la question de l'apprentissage sur le devant de la scène, puisqu'il est supposé qu'une représentation se forme et se stabilise par la modification du nombre et de la force des liens entre les unités qui la composent. On rejoint ainsi la conception de l'apprentissage énoncée par Hebb (1949). Cette modification se produit lorsque l'apprenant constate un phénomène nouveau par rapport à la représentation en question, soit au niveau du stimulus perçu, soit au niveau du feedback obtenu à la réponse. Par exemple, le concept LAUGH sera activé à partir du stimulus sonore standard /la:f/, mais un jour il sera constaté que le stimulus /laef/ désigne le même concept. Afin de tenir compte de cette possibilité, un nouveau lien d'excitation se forme dans la représentation lexicale, tel que l'allophone/ae/ peut également contribuer à l'activation du concept. Pour d'autres représentations en revanche, ce choix sera exclu et les représentations phonologiques devront entretenir des liens d'inhibition de manière à pouvoir distinguer, par exemple, HAM et HARM. Dans ce cas, la perception de /ae/ inhibera l'activation de HARM mais contribuera, par un lien d'excitation, à celle de HAM. Il ressort ainsi qu'une représentation lexicale est composée de trois réseaux interconnectés de manière complexe: orthographique, phonologique, sémantique. Si les deux premiers réseaux se stabilisent assez rapidement, il est clair que le niveau sémantique peut, en théorie, évoluer en permanence, surtout en L2 où il s'agit souvent d'apprendre un nouveau découpage conceptuel du monde.

5 Le connexionnisme fournit un cadre théorique intéressant non seulement parce que l'on y trouve une théorie de l'apprentissage mais aussi parce que celle-ci est susceptible d'être compatible avec des données neurophysiologiques issues du premier niveau d'analyse. Ce deuxième aspect est toutefois loin d'être démontré de manière convaincante, et pour un certain courant connexionniste ne constitue pas d'ailleurs l'objectif principal. Ainsi, pour 
ne prendre qu'un exemple, le rôle que jouent les neuromédiateurs n'est guère pris en compte lors des simulations informatiques connexionnistes. Il faut souligner par ailleurs que l'approche entière suscite de vives critiques parmi des cognitivistes classiques (Fodor 1995; Pinker \& Prince 1988). Il est probable que ce débat se résolve par une reconnaissance que les deux types de raisonnement peuvent coexister, le connexionisme au niveau subsymbolique et le cognitivisme classique au niveau symbolique (Smolensky 1988). Dans le même esprit, Neely (1991) remarque qu'il n'est pas nécessaire de raisonner en termes connexionnistes dans la mesure où la multiplicité d'unités et de liens mise en avant par cette théorie peut être considérée à un autre niveau comme une seule unité, la représentation lexicale. De ce point de vue, le lexique mental peut également être décrit comme un réseau de nœuds interconnectés, chaque nœud représentant une unité lexicale. Mais même si l'on préfère raisonner en termes de représentations locales et non pas distribuées, il reste toutefois à ne pas perdre de vue le processus de formation et d'ajustement des liens à l'intérieur de ces unités lexicales, processus d'apprentissage qui mène précisément à la constitution du réseau lexical. Par ailleurs, il est clair que la simulation connexionniste de certains comportements humains en matière d'apprentissage et d'accès lexical nous renseigne utilement sur le type de processus qui pourraient être à l'œuvre (Bechtel \& Abrahamsen 1991).

\section{Le réseau lexical $L 1$}

6 Parmi les trois domaines d'une représentation lexicale, c'est le domaine sémantique qui reste le plus ouvert à l'établissement de liens avec d'autres représentations. Des liens existent également sur la base de ressemblances orthographiques et phonologiques (Brown, Sharma \& Kirsner 1984 ; Grainger 1993 ; Meara 1983), mais le lexique mental est plus clairement organisé selon des rapports sémantiques. Cette organisation peut être conceptualisée sous forme d'un réseau de nœuds (Collins \& Loftus 1975), ou, suivant l'approche connexionniste, en mettant l'accent sur la distribution des représentations à travers une multiplicité de microtraits sémantiques (McRae \& Boisvert 1998). Lorsque deux termes sont sémantiquement proches, la perception d'un des termes facilite la reconnaissance du deuxième. Cette facilitation est mise en évidence par une diversité de tâches, dont la plus fréquente est la décision lexicale. Il s'agit de décider si une suite de lettres apparaissant à l'écran d'un ordinateur constitue un mot (par exemple, CHAT) ou non (par exemple CHAL). Dans le cas d'un vrai mot, la réponse est plus rapide si le mot est précédé d'un mot sémantiquement relié (par exemple CHIEN). Pour expliquer ce phénomène, on postule l'existence de liens entre les deux représentations, qui peuvent varier en force ou en nombre, et d'une diffusion automatique d'activation, de sorte que la représentation du deuxième mot présenté (la cible) est préactivée par le premier mot (l'amorce). Les notions de nombre et de force des liens ne recouvrent pas exactement la même réalité mais peuvent mener au même résultat. Un nombre élevé de liens suppose qu'il existe plusieurs traits sémantiques en commun et que les deux termes sont étroitement liés grâce à ce chevauchement conceptuel. Qu'un lien soit fort met davantage l'accent sur un lien direct entre nœuds conceptuels, fournissant une voie que peut emprunter l'activation de manière rapide, voire automatique.

7 CHIEN et CHAT sont reliés sémantiquement par l'existence d'un certain nombre de traits qu'ils possèdent en commun. Parmi ces traits, certains sont plus saillants que d'autres; en l'occurrence le trait <animal domestique> a un pouvoir définissant plus fort que les 
autres. L'existence d'un chevauchement conceptuel favorise la création d'un autre lien, de similitude, directement reliant les deux concepts (figure 1).

Lorsque le chevauchement conceptuel atteint un degré élevé, on pourra parler de synonymie, un des plus puissants principes organisateurs du lexique mental (Miller \& Fellbaum, 1991). En même temps, cependant, il est nécessaire de distinguer entre deux concepts, et de ce fait CHIEN et CHAT entretiennent des rapports d'inhibition avec les traits <miaule> et <aboie> respectivement. Bien qu'il soit d'une complexité accrue par rapport aux deux autres domaines, on retrouve ainsi dans le domaine sémantique une organisation en réseau semblable à ce qui est constaté pour les représentations orthographique et phonologique. Ces réseaux se forment grâce au constat de régularités statistiques, sur le plan formel et conceptuel, d'où ressortent des représentations définies en termes de ressemblances, de différences et de rapports fonctionnels.

Figure 1. Création d'un lien de synonymie/ressemblance grâce à des traits conceptuels partagés



Parmi les rapports les plus étudiés en première langue se trouvent la synonymie, l'antonymie (Beauvillain \& Ségui 1983; Scampa 1986; Warren 1977), et l'hyperonomie (Rosch 1975 ; Becker 1980). Ces étiquettes désignent des catégories plus ou moins précises mais restent commodes car elles regroupent de nombreux items lexicaux. Il en existe cependant beaucoup d'autres, moins souvent explicités, qu'il conviendrait également d'éclaircir. Si la lexicographie fournit des exemples de formalisation de ces rapports (Mel'cuk 1984), il reste à déterminer leur importance, et les raisons éventuelles de celleci, sur le plan psycholinguistique. Une distinction est souvent faite entre rapport sémantique et rapport associatif (Fischler 1977 ; Shelton \& Martin 1992). Ainsi SOURIS et FROMAGE n'ont pas de chevauchement conceptuel mais sont reliés par association. Cette association revêt toutefois un caractère sémantique si l'on considère que son amour immodéré du fromage est un trait à fort pouvoir définissant du concept SOURIS. Autrement dit, la force de ce lien, unique mais saillant, compense le manque des multiples liens établis lorsque plusieurs traits sémantiques sont partagés. L'existence de traits sémantiques partagés ne constitue pas d'ailleurs une condition suffisante pour observer un effet de facilitation entre deux concepts. Par exemple, malgré leur chevauchement conceptuel, la perception préalable de PAIN ne facilite pas l'identification de GATEAU (Shelton \& Martin 1992; voir aussi McRae \& Boisvert 1998). Les raisons précises de ce type de résultat restent à déterminer, mais il est clair que la force des liens joue un rôle prépondérant. Le pouvoir définissant associé ici à la notion de force résulte de l'attribution conventionnelle de traits à un concept, sur la base d'une expérience commune, ou perçue comme telle, du référent désigné. 


\section{La constitution d'un réseau $\mathrm{L} 2$}

10 La différence majeure entre L1 et L2 vient du fait qu'en L2 il s'agit d'apprendre une nouvelle forme lexicale pour un concept qui est déjà en place. Par ailleurs, ce processus doit souvent s'accompagner d'un réaménagement du concept de manière à prendre en compte les rapports différents entre concept et mot dans les deux langues. Le mot français échelle correspond à deux mots anglais, ladder et scale, alors que le mot anglais ball correspond à deux mots français, balle et ballon. Si ces exemples s'appuient sur des référents perceptuels relativement faciles à distinguer, il existe de nombreux cas plus difficiles à saisir, par exemple la série déjà, encore, toujours, jamais et leurs homologues, mais non équivalents, already, still, always, yet, ever, never.

11 Ces deux apprentissages, de la forme et du sens, suivent des rythmes différents. Pour un apprenant $\mathrm{L} 2$, une hypothèse plausible au départ est que le concept sous-jacent à un mot L2 est le même que pour sa traduction L1 (McWhinney 1997), même au prix de choisir une traduction erronée parmi plusieurs possibilités. L'attention porte alors sur la forme dont la reconnaissance rapide est une exigence, ainsi que le note Yang (1997) : «The development of automated word recognition may be an important prerequisite for the development of automated semantic activation. » Pour des mots fréquents, rencontrés tôt dans l'apprentissage, il se crée cependant des liens entre mot et concept assez rapidement, tel que le premier active automatiquement le second sans passage obligé par L1 (Altarriba \& Mathis 1997; Chen 1990). On pourra parler alors de médiation conceptuelle pour L2 de la même manière que pour L1. Ce phénomène pourrait se produire après une demi-douzaine de rencontres avec un mot; de surcroit, la médiation conceptuelle pour L2 se met en place aussi rapidement, que la méthode d'apprentissage soit par images ou par traductions (Chen 1990).

Il importe à ce stade de préciser ce que l'on entend par concept. Suivant Saussure (1916), il est supposé que le sens d'un mot comporte à la fois un aspect référentiel, à savoir la représentation perceptuelle du phénomène désigné par le mot, et un aspect différentiel, dépendant du sens des mots conceptuellement reliés, qui lui confèrent sa valeur sémantique. C'est ce deuxième aspect qui se met en place avec la constitution d'un réseau L2. Autrement dit, une partie du sens d'un mot provient de la connaissance que nous avons des rapports de ce mot avec d'autres mots de la langue, ses voisins sémantiques. Il est à noter que parmi ces rapports comptent les collocations (Firth 1957; Yorio 1980 ; Frenck-Mestre \& Prince 1997), qui contribuent également au sens.

13 Au stade initial de l'apprentissage, par le biais d'un transfert positif, l'apprenant peut constituer un réseau selon les mêmes types de liens sémantiques qui fonctionnent en L1 (Prince \& Frenck-Mestre 1999). Cependant, il s'agit davantage de miniréseaux distincts, formés de quelques mots fortement reliés (par exemple, des noms de parties du corps, de couleurs, ou de parenté familiale) que d'un réseau fonctionnellement autonome. Pour que celui-ci se constitue, il faut, d'une part, que des éléments syntaxiques se mettent également en place, et d'autre part que le lexique s'étoffe par l'addition d'autres mots reliés. Parmi ces liens, les collocations revêtent une grande importance dans la mesure où elles diffèrent souvent dans les deux langues, et forment ainsi un indice précieux sur la valeur sémantique précise des mots L2. Tant qu'un mot L2 reste sémantiquement isolé, son lien le plus fort sera avec son équivalent de traduction en L1. L'ensemble du réseau L2 émergeant comporterait donc plusieurs miniréseaux plus ou moins complets, dont les 
rapports avec L1 seront déterminés par la stabilité et la force des liens internes à chaque miniréseau (figure 2). Il est probable, d'ailleurs, que ce type d'organisation persiste longtemps pendant l'apprentissage, mais que ses carences deviennent moins visibles grâce à l'accroissement du vocabulaire et de la compétence communicative.

Une question largement débattue dans la littérature (Kroll \& Stewart 1994; La Heij et al., 1996 ; De Groot et al. 1994), concerne une éventuelle asymétrie dans les rapports L1 - L2, telle qu'un mot L2 serait relié à son équivalent de traduction par un lien fort au niveau lexical (association lexicale), alors qu'un mot L1 serait relié plus fortement au concept sous-jacent (médiation conceptuelle). Si une partie du niveau conceptuel dépend de la connaissance des mots qui composent le miniréseau, une organisation lexicale comme celle de la figure 2 montre qu'une structure symétrique et asymétrique peut coexister chez le même individu (De Groot \& Hoeks 1995 ; Prince 1998).

Figure 2. Schéma d'un réseau lexical L1 et d'un réseau L2 émergeant

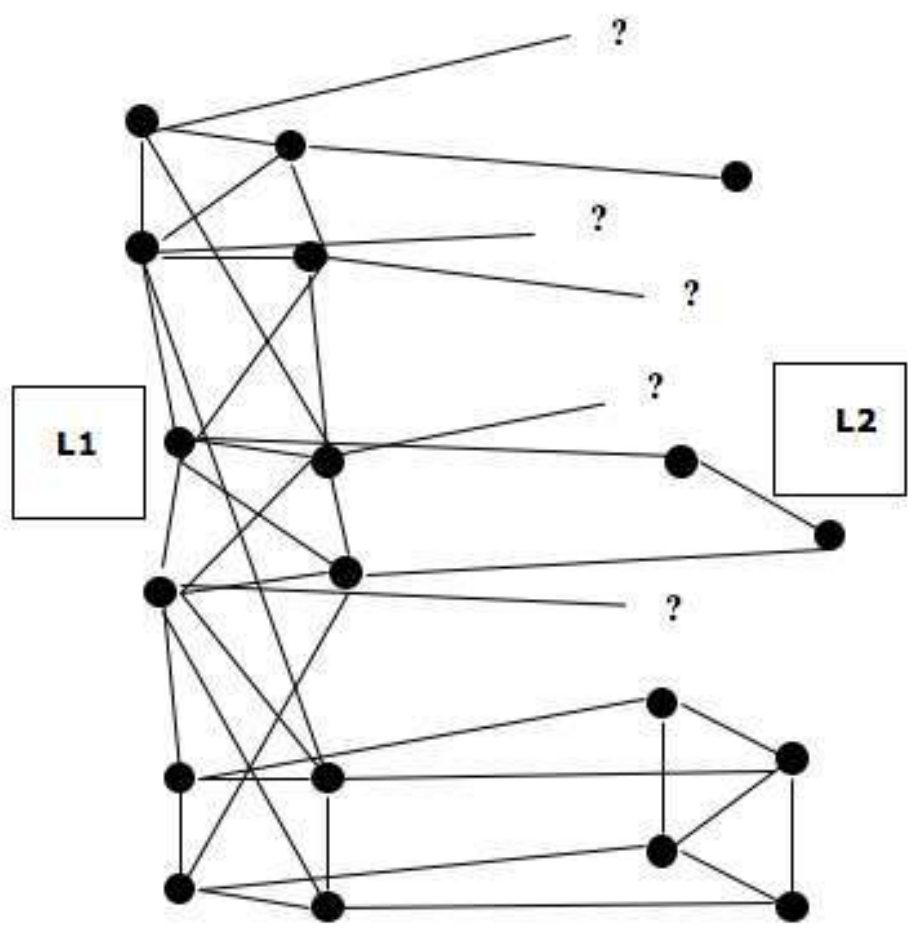

\section{Doit-on enseigner le vocabulaire?}

Suivant des recherches effectuées en L1 (Nagy, Herman \& Anderson 1985 ; Nation \& Coady 1988), il est parfois estimé que la meilleure façon d'augmenter son vocabulaire L2 consiste à lire beaucoup, non pas pour analyser les formes linguistiques, mais pour accéder au sens du texte, ou simplement pour le plaisir (Krashen 1989). Il est supposé ainsi que grâce à la rencontre de nouveaux mots plusieurs fois dans des contextes différents, ils s'insèrent dans le réseau L2 de manière inconsciente. Pour Krashen (1989), cette idée vient à l'appui de son hypothèse de l'input, selon laquelle une deuxième langue s'acquiert par le biais d'une exposition à une quantité suffisante d'input compréhensible, que l'apprenant traite pour accéder au sens (Krashen 1987). Selon cette conception de 
l'apprentissage, il n'est pas nécessaire de planifier un enseignement lexical spécifique, puisque les textes eux-mêmes sont source de nouveaux mots, dont l'apprentissage découlera sans effort à partir du moment où ils apparaissent plusieurs fois.

Plusieurs données de la psychologie cognitive viennent conforter cette idée. La rapidité de la reconnaissance visuelle des mots est fonction de leur fréquence dans la langue (Scarborough et al. 1977). Ceci n'est pas en soi un argument en faveur de la position de Krashen, mais il semble raisonnable de laisser des textes authentiques déterminer la fréquence des rencontres. Pour Hasher et Zacks (1979), l'encodage de la fréquence d'occurrence d'un phénomène est un processus inconscient, ce qui signifie que certains aspects, ne serait-ce que formels, sont également traités de manière inconsciente. Autrement dit, avant d'encoder la fréquence d'un phénomène, il faut déjà qu'il soit perçu comme tel (Johnson et al. 1989), ce qui dans le cas d'un mot, suppose au moins une reconnaissance de la forme. Bien entendu, sur le plan lexical, cette reconnaissance se fait plus facilement que sur le plan syntaxique, et il est probable que pendant la lecture les mots inconnus sont traités non seulement sur le plan formel, mais aussi, au moins partiellement, sur le plan sémantique. On peut ainsi expliquer la formation rapide de miniréseaux L2 et la mise en place d'une médiation conceptuelle L2. Au regard des résultats de Chen (1990), De Groot (1995) avance l'idée que la pédagogie n'a que peu d'effet sur l'apprentissage des mots, car la médiation conceptuelle L2 se met en place quelle que soit la méthode d'apprentissage adoptée. Sur le plan théorique, cette idée est compatible avec l'apprentissage tel que le connexionnisme l'envisage, puisque le nombre et la force des liens qui donnent sa cohésion à la représentation mentale des mots se modifient, dans le sens d'une plus grande stabilité et d'une plus grande rapidité d'accès, à chaque rencontre. Tous ces éléments plaident en faveur d'une acquisition inconsciente des mots à travers la lecture extensive.

17 Cependant, malgré les bienfaits indéniables de la lecture, il n'est pas communément admis qu'elle soit suffisante pour garantir un accroissement du lexique. En dehors du fait que cette approche suppose un degré élevé de motivation, il est constaté que le traitement sémantique des mots inconnus est souvent approximatif, voire inexact (Hulstijn 1992), et que les progrès sont lents et aléatoires (Paribakht \& Wesche 1997). Par ailleurs, il est probable qu'un certain niveau de compétence L2 soit nécessaire avant que l'apprenant puisse bénéficier pleinement de l'activité de lecture (Coady 1997). Enfin, la consolidation du réseau L2 est laissée au hasard puisqu'aucun rebrassage systématique n'est prévu.

18 Ces remarques ne signifient pas que l'apprentissage conscient de listes de vocabulaire soit le meilleur remède. Bien que cette forme d'apprentissage lexicale semble facile (Nation 1982), l'utilisation dans des contextes L2 appropriés des mots appris ainsi s'avère difficile (Prince 1996). Il est plutôt recommandé de mettre en place des activités spécifiques au lexique qui tiennent compte du contexte L2 (Nation \& Newton 1997 ; Lewis 1993). Sans passer en revue ici toute la gamme des activités proposées, il est possible de noter quelques principes qu'il serait utile de respecter dans un programme d'enseignement lexical.

19 Si un nombre suffisant de rencontres avec les mots peut être assez facilement atteint, il est moins aisé de satisfaire à l'exigence d'un espacement optimal de celles-ci. Groupées au départ, ces rencontres pourront, voire devront, s'espacer davantage dans le temps pour garantir l'affermissement de la représentation mentale (Gairns \& Redman 1986). 

prendre ici au sens large, comme tout ce qui entoure un mot lors de sa présentation. Le contexte linguistique peut consister en un seul mot, relié ou non au mot à apprendre, ou un segment plus long (phrase ou paragraphe). Le contexte cognitif dépend de la tâche à effectuer. Des décisions au niveau de la forme, orthographique ou phonologique, peuvent être bénéfiques, mais pour que la représentation sémantique s'affine, les tâches devront surtout faire intervenir divers types de traitement sémantique : jugements d'associations selon les différentes possibilités sémantiques et collocationnelles, tâches de reconnaissance et de production, tâches auditives et visuelles.

Le critère de la fréquence des mots dans la langue reste un bon indice pour le choix des mots à enseigner. Selon Nation et Coady (1988), un apprenant exploite pleinement un contexte lorsqu'il connaît $98 \%$ des mots qui composent le texte. Son besoin immédiat est donc l'apprentissage des mots les plus fréquents de la langue. Nation \& Newton (1997) affirment l'importance des 2000 mots les plus fréquents, qui représente $87 \%$ de la langue écrite. problème de l'interférence, que celle-ci provienne de L2 ou de L1. Higa (1963) met en garde contre des activités d'association sémantique en soulignant qu'elles sont souvent source de confusion. Le réseau L2 est en effet fragile tant que les représentations mentales des mots qui le composent ne sont pas précises et rapidement accessibles. À cet égard, la contrainte est double et contradictoire : d'une part il ne faudrait rajouter un mot au lexique que lorsque les représentations lexicales déjà établies sont suffisamment précises; d'autre part, ces représentations ne sont précises que lorsque leurs voisins sémantiques sont connus. Une interférence se produit lorsque les représentations ne sont pas assez fortement établies, ou que leur valeur sémantique n'est pas précisément délimitée. Pour distinguer, par exemple entre les expressions anglaises pour Il fait du bon travail et Il a un bon travail, l'apprenant doit connaître les valeurs sémantico-syntaxiques de work et job. Sur le plan formel, work est également associé à walk, qui fournit donc une autre possibilité d'erreur. Enfin, en passant cette fois par L1, la ressemblance entre travail et travel explique des erreurs comme He's got a good travel. Ces confusions sont possibles tant que les traits par lesquels ces mots sont différenciés ne sont pas assez solidement établis pour permettre une diffusion d'activation selon des liens d'excitation et d'inhibition appropriés. En ce qui concerne un programme d'enseignement lexical, cette contrainte est mieux prise en compte par un compromis : une intégration graduelle mais régulière des mots pour favoriser une restructuration concomitante des concepts, en retardant l'introduction de nouvelles formes jusqu'à ce que la reconnaissance de formes connues soit en voie d'automatisation.

Enfin il peut être intéressant de travailler sur la vitesse de l'accès lexical, car un bon lecteur est celui chez qui, entre autres, l'accès lexical est rapide. Ainsi des activités pilotées par ordinateur, où l'apprenant est informé de ses temps de réponse et ses erreurs, sont un bon moyen de combiner automatisation de formes connues et intégration de formes nouvelles, au moins jusqu'au stade où la lecture devient moins laborieuse et plus profitable. 


\section{Conclusion}

En partant du substrat anatomique, composé de réseaux de neurones, nous avons tenté ici de montrer la pertinence de la notion de réseau appliqué au lexique mental. Lorsque l'on adopte une analyse de ce type, il devient difficile d'opérer une distinction nette entre processus et représentation (Durgunoglu \& Roediger 1987). La manière dont l'activation se diffuse à travers l'ensemble des unités qui constituent une représentation dépend des conditions de présentation du stimulus et de la tâche à effectuer. Une représentation lexicale, tout en restant ouverte à la création d'autres liens, se stabilise néanmoins lorsque la réponse obtenue est conforme aux attentes du locuteur, plusieurs fois de suite. En production, cette réponse est fournie par l'interlocuteur, qui peut, si le message n'est pas clair, entamer un processus de négociation de sens. En compréhension, la réponse est fournie par l'interaction entre le lecteur et le texte, où le lecteur est amené à juger de la cohérence sémantique de l'ensemble, résultat d'un processus d'intégration sémanticosyntaxique des mots individuels tels qu'il se les représente.

Une représentation lexicale peut ainsi être caractérisée comme un ensemble de théories et d'attentes (Hataigandi 1989), et les liens qui la déterminent se modifient, en force et en nombre, lorsqu'un écart est constaté entre ces attentes et la réponse obtenue. Chez le locuteur adulte natif, une représentation lexicale correspond à une norme descriptive socialement établie, mais toujours ouverte à négociation. La même représentation chez un apprenant subit de nombreuses évolutions avant d'atteindre cette norme. Cette évolution se traduit par la modification des liens qui se forment et s'ajustent en fonction des indices perçus dans l'environnement. La constitution d'un réseau L2 procède ainsi par tâtonnements, avec beaucoup d'approximations et d'erreurs, jusqu'à ce qu'il permette une autonomie fonctionnelle grâce à sa taille, sa précision, et sa rapidité d'accès.

\section{BIBLIOGRAPHIE}

Altarriba, J. \& K.M. Mathis. 1997. « Conceptual and lexical development in second language acquisition ». Journal of Memory and Language 36, 550-568.

Beauvillain, C. \& J. Ségui. 1983. «Rôle du contexte dans la décision lexicale : Rapidité d'établissement d'une facilitation sémantique ». L'Année psychologique 83, 39-52.

Bechtel, W. \& A. Abrahamsen. 1991. Connectionism and the Mind: An introduction to parallel processing in networks. Oxford : Blackwell.

Becker, C.A. 1980. « Semantic context effects in visual word recognition: An analysis of semantic strategies ». Memory and Cognition 8, 493-512.

Bierwisch, M. 1970. « Semantics ». In Lyons, J. (dir.), New Horizons in Linguistics. Harmondsworth : Penguin Books, 166-184. 
Brown, H., Sharma, N.K . \& K . Kirsner. 1984. «The role of script and phonology in lexical representation ». Quarterly Journal of Experimental Psychology 36A, 491-505.

Chen, H.C. 1990. « Semantic facilitation and translation priming effects in Chinese-English bilinguals », Memory and Cognition, 18, 279-288.

Coady, J. 1997. « L2 vocabulary acquisition through extensive reading ». In Coady, J. \& T Huckin (dir.), Second Language Vocabulary Acquisition. Cambridge : Cambridge University Press, 225-237.

Collins, A.M. \& E.F. Loftus. 1975. « A spreading-activation theory of semantic processing ». Psychological Review 82/6, 407-428.

De Groot, A.M.B. 1992. «Bilingual lexical representation: A closer look at conceptual representations ». In R. Frost \& L. Katz (dir.), Orthography, Phonology, Morphology and Meaning. Amsterdam : Elsevier, 389-412.

De Groot, A.M.B. 1995. « Determinants of bilingual lexicosemantic organisation ». Computer Assisted Language Learning 8, 151-180.

De Groot, A.M.B., Dannenburg, L. \& J.G. Van Hell. 1994. « Forward and backward word translation ». Journal of Memory and Language 33, 600-629.

De Groot, A.M.B. \& J. Hoeks. 1995. « The development of bilingual memory: Evidence from word translation by trilinguals ». Language Learning 45/4, 683-724.

Durgunoglu, A.Y. \& H.L. Roediger. 1987. « Test differences in accessing bilingual memory ». Journal of Memory and Language 26, 377-391.

Firth, J.R. 1957. Papers in Linguistics 1934-1951. Oxford : Oxford University Press.

Fischler, I. 1977. « Semantic facilitation without association in a lexical decision task ». Memory and Cognition 5, 335-339.

Fodor, J.A. 1995. « The folly of simulation ». In Baumgartner \& Payr (dir.), Speaking Minds. Princeton, NJ : Princeton University Press.

Frenck-Mestre, C. \& P. Prince. 1997. « Second language autonomy ». Journal of Memory and Language 37, 481-501.

Frenck-Mestre, C. \& P. Prince. 1999. «La compréhension écrite d'une deuxième langue : lexique et syntaxe ». Langues Modernes 3, 58-69.

Gairns, R. \& S. Redman. 1986. Working with Words: A guide to teaching and learning vocabulary. Cambridge : Cambridge University Press.

Grainger, J. 1993. «Visual word recognition in bilinguals ». In Screuder \& Weltens (dir.), The Bilingual Lexicon. Amsterdam : John Benjamins, 11-25.

Hattiangadi, J.N. 1987. How is language possible? La Salle : Open Court.

Hasher, L. \& R.T. Zacks. 1979. « Automatic and effortful processes in memory ». Journal of Experimental Psychology: General 108, 356-388.

Hebb, D.O. 1949. The Organization of Behavior: A neuropsychological theory. New York : Wiley.

Higa, M. 1963. « Interference effects of intralist word relationships in verbal learning ». Journal of Verbal Learning and Verbal Behavior 2, 170-175.

Hulstijn, J.H. 1992. « Retention of inferred and given meanings: Experiments in incidental vocabulary learning ». In Arnaud \& Béjoint (dir.), Vocabulary and Applied Linguistics. Londres : Macmillan. 
Johnson, M.K et al. « Frequency judgments : The problem of defining a perceptual event », Journal of Experimental Psychology : Learning, Memory and Cognition, 15, 126-136, 1989.

Krashen, S. 1987. Principles and Practice in Second Language Acquisition. Londres : Prentice Hall.

Krashen, S. 1989. « We acquire vocabulary and spelling by reading: Additional evidence for the input hypothesis ». Modern Language Journal 73, 440-464.

Kroll, J.F. \& E. Stewart. 1994. « Category interference in translation and picture naming: Evidence for asymmetric connections between bilingual memory representations ». Journal of Memory and Language $33,149-174$.

La Heij, W. et al. 1996. « Nonverbal context effects in forward and backward translation: Evidence for concept mediation ». Journal of Memory and Language 35, 648-665.

Lewis, M. 1993. The lexical Approach: The state of ELT and a way forward. Hove, England : Language Teaching Publications.

Marr, D. 1982. Vision. San Fransisco : Freeman.

McRae, K. \& S. Boisvert. 1998. « Automatic semantic similarity priming ». Journal of Experimental Psychology: Learning, Memory and Cognition 24, 558-572.

McWhinney, B. 1997. « Second language acquisition and the competition model ». In De Groot \& Kroll (dir.), Tutorials in Bilingualism. Mahwah, NJ : Lawrence Erlbaum, 113-142.

Meara, P. 1983. « Word associations in a foreign language ». Nottingham Linguistics Circular 11/2, 19-38.

Mel'cuk, I. 1984. Dictionnaire explicatif et combinatoire du français contemporaine. Montréal : University of Montreal Press.

Miller, G.A. \& C. Fellbaum. 1991. « Semantic networks of English ». Cognition 41, 197-229.

Monsell, S. 1991. "The nature and locus of word frequency effects in reading ». In Besner \& Humphreys (dir.), Basic Processes in Reading: Visual word recognition. Hillsdale, NJ : Lawrence Erlbaum, 148-197.

Nagy, W., Herman, P. \& R. Anderson. 1985. « Learning words from context ». Reading Research Quarterly 20, 233-253.

Nation, I.S.P. 1982. « Beginning to learn foreign vocabulary: A review of the research ». RELC Journal 13, 14-36.

Nation, I.S.P. \& J. Coady. 1988. « Vocabulary and reading ». In Carter \& McCarthy (dir.), Vocabulary and Language Teaching. Londres : Longman, 97-110.

Nation, I.S.P. \& J. Newton. 1997. « Teaching vocabulary ». In Coady, J. \& T Huckin (dir.), Second Language Vocabulary Acquisition. Cambridge : Cambridge University Press, 238-254.

Neely, J.H. 1991. « Semantic priming effects in visual word recognition: A selective review of current findings and theories ». In Besner \& Humphreys (dir.), Basic processes in reading: Visual word recognition. Hillsdale, NJ : Lawrence Erlbaum, 264-336.

Paribakht, T.S. \& M. Wesche. 1997. « Vocabulary enhancement activities and reading for meaning in second language vocabulary acquisition ». In Coady, J. \& T Huckin (dir.), Second Language Vocabulary Acquisition. Cambridge : Cambridge University Press, 174-200.

Pinker, S. \& A. Prince. 1988. « On language and connectionism: Analysis of a parallel distributed processing model of language acquisition ». Cognition 28, 73-193. 
Prince, P.W.J. 1996. « Second language vocabulary learning: The role of context versus translation as a function of proficiency ». Modern Language Journal 80, 478-493.

Prince, P.W.J. 1998. «L'effet d'asymétrie dans le traitement lexical bilingue ». Psychologie française 43-4, 283-296.

Rosch, E. 1975. « Cognitive representations of semantic categories ». Journal of Experimental Psychology: General 104, 192-233.

Rose, S. 1993. The Making of Memory. Londres : Bantam Press.

Rumelhart, D.E., McClelland, J.L. \& the PDP Research Group. 1986. Parallel Distributed Processing: Explorations in the microstructure of cognition. Vol. Foundations. Cambridge, MA : MIT Press/ Bradford Books.

Saussure, F. de. 1916. Cours de linguistique générale (C. Bally \& A. Sechehaye, dir.). Lausanne : Payot (réédité à Paris).

Scampa, P. 1986. «Effet de synonymie dans une tâche de décision lexicale ». Bulletin de Psychologie $34,451-455$.

Scarborough, D.L., Cortese, C. \& H.S. Scarborough. 1977. « Frequency and repetition effects in lexical memory ». Journal of Experimental Psychology : Human Perception and Performance 3, 1-17.

Shelton, J.R. \& R.C. Martin. 1992. « How semantic is automatic semantic priming? ». Journal of Experimental Psychology 18, 1191-1210.

Smolensky, P. 1988. « On the proper treatment of connectionism ». Behavioral and Brain Sciences 11, 1-74.

Warren, R.E. 1977. « Time and spread of activation in memory ». Journal of Experimental Psychology: Human Memory and Learning 3, 458-466.

Yang, L. 1997. « Tracking the acquisition of L2 vocabulary: The Keki language experiment ». In Coady, J. \& T Huckin (dir.), Second Language Vocabulary Acquisition. Cambridge : Cambridge University Press, 125-155.

\section{RÉSUMÉS}

En partant de la prise en compte de la dimension physiologique de l'apprentissage, le présent article explore la notion de réseau telle qu'elle s'applique à l'apprentissage lexical en deuxième langue. Conformément à la plupart des théories psycholinguistiques, y compris le connexionnisme, on supposera que les représentations mentales s'établissent et deviennent accessibles grâce à la diffusion d'une activation à travers des populations de neurones. En gardant cette idée en tête lors de l'établissement d'un modèle fonctionnel des phénomènes cognitifs, on peut tenter de caractériser le développement du lexique mental en deuxième langue. Certains aspects de ce développement sont automatiques, variant selon le degré et la nature des contacts avec la langue cible. D'autres peuvent être infléchis par une pédagogie appropriée, c'est-à-dire qui prend en compte non seulement les objectifs à atteindre, mais aussi les contraintes imposées par le fonctionnement cognitif des apprenants.

Beginning with a consideration of the physiological dimension of learning, this article explores the notion of network as it applies to second language vocabulary learning. In common with most psycholinguistic theories, notably connectionism, it is assumed that mental representations are established and become accessible thanks to the spread of activation through populations of 
neurones. Bearing in mind this idea when drawing up a functional model of cognitive processing, a description of the way a second language mental lexicon develops may be attempted. Certain aspects of this development are automatic, varying according to the degree and nature of exposure to the target language. Others may be influenced by appropriate teaching methods, namely those that take into account not only the goals to be achieved but also the constraints imposed by learners' cognitive functioning.

INDEX

Keywords : activation, learning, lexical representation, mental lexicon, network

Mots-clés : activation, apprentissage, lexique mental, représentation lexicale, réseau

\section{AUTEUR}

\section{PETER PRINCE}

Peter Prince est maître de conférences à l'université d'Aix-Marseille 1, où il conduit ses recherches au sein du CREPCO, et dirige le CALUP (Centre d'Apprentissage des Langues de l'Université de Provence). Peter.Prince@univ-provence.fr 